\title{
A Supervising System for Robot Application in Surgery
}

\author{
Dirk Engel, Joerg Raczkowsky, Heinz Woern \\ Institute for Process Control and Robotics, Universität Karlsruhe (TH) \\ Kaiserstraße 12, 76128 Karlsruhe, Germany \\ dEngel@ira.uka.de | rkowsky@ira.uka.de | woern@ira.uka.de
}

\begin{abstract}
In this paper a supervising system for robot application in surgery is described. Since a robot in surgery has to meet special safety requirements the robot application has to be controlled not only by the responsible surgeon but also by redundant sensors. Therefore our robot system for bone repositionings in craniofacial surgery is equipped with internal and external sensors. This paper discusses the use of an infrared navigation system and a force-torquesensor as supervising sensors.
\end{abstract}

\section{Introduction}

The fundamental issue of robots in surgery is the safety of the involved persons: patient, physicians and nurses, for example. Therefore a robust and redundant controlling system is required, in order to be resistant against failure. In craniofacial surgery bone repositionings are carried out. Due its closeness to vital parts, i.e. the brain, high precision and safety is indispensable. Hence, the robot is intended for supporting the surgeon drilling and milling the skull bone 1 While the robot moves along the preoperatively planned trajectory the surgeon authorizes every robot movement by pressing the dead-man switch. Additionally, the robot is supervised by an infrared navigation system (INS) and a force-/torque sensor (FTS). This work is funded by the German Research Foundation (DFG), as it is part of the special research program SFB 414.

\section{System Architecture and Supervising Sensors}

The system consists of two computers, the robot controller and a PC for sensor data acquisition and processing. Both computers are connected via a RS232 serial line. Further, the PC accesses a digital input signal and the emergency-stop-circuit of the robot controller via a relais card. The digital signal provides the possibility of a socalled soft interrupt; switching logical high to logical low signal causes the robot to move its tool 25 millimeter back from the current location. Such a way the capability of moving the milling cutter out of the bone in the event of failure is retained. Accessing the emergency-circuit, so-called hard interrupt, causes an immediate robot stop.

A rigid body equipped with IR-LEDs which can be tracked by the INS is mounted to the robot tool and is also fixed to the patients head. The INS supervises the 
movements of the robot rigid body relative to the patient rigid body. Such a way the INS is able to detect shortcomings of the patient fixation and wrong robot movements, additionally. Computing the relative position brings in the measuring errors of both rigid bodies. Further, the tracked positions do not coincide with the origin of the planning data and robot tool tip position, respectively. Therefore the measured positions have to be transformed into these locations. Transforming the tracked robot tool position into the tool tip position causes an intensification of the measured orientation inaccuracies. An orientation error of $\varphi=1^{\circ}$ yields a presumable tool tip positioning error of about $\mathrm{d}=3.5 \mathrm{~mm}$, if the distance between rigid body and tool tip is $1=20 \mathrm{~cm}: \mathrm{d}=1 * \tan \varphi$. For that reason not only the measured absolute positioning error is supervised, but two types of errors are distinguished. First, the computed distance $\mathrm{d}_{\mathrm{z}}$ between tool tip and trajectory measured along the tool axis ( $\mathrm{z}$ axis) and, second, $d_{y}$ whereby $y=z \times x$ and $x$ is the direction of the trajectory. An error is detected on considering both errors.

The FTS controls the forces and torques affected to the robot tool. It can be used to detect collisions of the robot tool with obstacles during service and approaching movements. Furthermore it is used to control the forces and torques during the milling/drilling process. Since this sensor provides a high data acquisition rate RTLinux 2 is used as real time operating system of the PC. The FTS software client program supervises the forces and torques regarding to the adjusted thresholds. In detail the real time kernel module monitors: the amount of each force/torque direction, the amount of the overall forces and torques, and the force/torque gradients. In case of exceeding an adjusted threshold, the client accesses the digital signal line or the robot emergency-stop-circuit. Fig. 1 depicts the recorded monitoring data of a trajectory milled out in four steps.
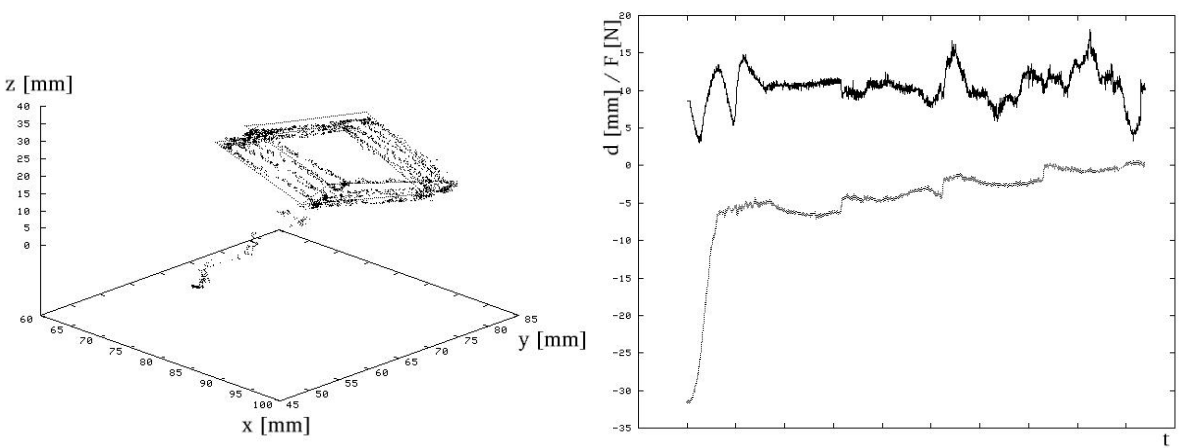

Fig. 1. Left: Trajectory supervised by INS. Right: detected distance $d_{z}$ to maximal cutting depth (gray) and corresponding overall force (black).

\section{References}

1. Engel, D., Raczkowsky, J., Woern, H.: "A Safe Robot System for Craniofacial Surgery", IEEE Inter. Conf. On Robotics and Automation, Seoul, Korea, 2001.

2. RTLinux: http://www.rtlinux.org, March 2001. 